\title{
Serum albumin is present at higher levels in alcoholic liver cirrhosis as compared to HCV-related cirrhosis
}

\author{
KAZUHIRO KOTOH ${ }^{1}$, MARIE FUKUSHIMA ${ }^{1}$, YUKI HORIKAWA ${ }^{1}$, SHINSAKU YAMASHITA ${ }^{1}$, \\ MOTOYUKI KOHJIMA ${ }^{2}$, MAKOTO NAKAMUTA ${ }^{2,3}$ and MUNECHIKA ENJOJI ${ }^{3,4}$ \\ ${ }^{1}$ Department of Hepatology and Pancreatology, Kyushu University Hospital, Fukuoka; \\ ${ }^{2}$ Department of Gastroenterology; ${ }^{3}$ Clinical Research Center, National Hospital Organization, \\ Kyushu Medical Center, Fukuoka; ${ }^{4}$ Health Care Center, Fukuoka University, Fukuoka, Japan
}

Received August 10, 2011; Accepted September 13, 2011

DOI: $10.3892 /$ etm.2011.370

\begin{abstract}
Residual hepatic functional reserve in cirrhotic patients is generally evaluated by a multivariate scoring system (Child-Pugh classification), which includes serum albumin levels as a variable. However, several patients show discrepancies between serum albumin levels and the progression of liver fibrosis, especially those with alcoholic cirrhosis. To assess whether hepatic capacity of protein synthesis varies with the etiology of cirrhosis, serum albumin and cholinesterase levels, and prothrombin time were compared between alcoholic cirrhosis and hepatitis $\mathrm{C}$ virus (HCV)-related cirrhosis. To minimize the influence of malnutrition and extrahepatic platelet destruction, patients with hepatocellular carcinoma, uncontrolled diabetes, appetite loss and/or splenal longitudinal size $>15 \mathrm{~cm}$ were excluded. The patients with compensated liver cirrhosis were divided into three groups as follows: alcohol ${ }^{+} / \mathrm{HCV}^{+}$(alcohol + $\mathrm{HCV}$ group; $\mathrm{n}=31$ ), alcohol ${ }^{-} /$ $\mathrm{HCV}^{+}\left(\mathrm{HCV}\right.$ group; $\mathrm{n}=31$ ) and alcohol ${ }^{+} / \mathrm{HCV}^{-}$(alcohol group; $\mathrm{n}=27$ ). These groups were adjusted with respect to age, gender, body mass index and platelet count. Serum albumin levels in the alcohol group were significantly higher than those in the $\mathrm{HCV}$ group, with a difference of approximately $0.5 \mathrm{~g} / \mathrm{dl}$ in every class of platelet count. The correlation of the alcohol + $\mathrm{HCV}$ group was intermediate between the alcohol and HCV groups. On the other hand, the correlations between serum cholinesterase levels and platelet counts were similar among the three groups. The prothrombin time was also comparable among the groups. Accordingly, serum albumin levels were higher in patients with alcoholic cirrhosis and alcohol
\end{abstract}

Correspondence to: Dr Munechika Enjoji, Health Care Center, Fukuoka University, 8-19-1 Nanakuma, Jonan-ku, Fukuoka 814-0180, Japan

E-mail: enjoji@adm.fukuoka-u.ac.jp

Abbreviations: BMI, body mass index; ChE, cholinesterase; GGT, $\gamma$-glutamyl transpeptidase; HCC, hepatocellular carcinoma; HCV, hepatitis $\mathrm{C}$ virus; ICG, indocyanine green; PT, prothrombin time

Key words: alcohol, cirrhosis, hepatitis C virus, alcoholic liver disease consumption should be carefully considered when evaluating hepatic functional reserve.

\section{Introduction}

Accurate assessment of residual hepatic functional reserve is indispensable for selecting an adequate treatment for patients with liver cirrhosis, particularly for those with liver tumors. Several tests have been proposed for determining residual liver function; however, no single marker is entirely reliable for predicting residual function, since hepatocytes possess a wide array of different functions $(1,2)$. Instead of using a single marker, scoring systems using several parameters have been developed for assessing hepatic functional reserve and stratifying the severity of liver cirrhosis. Currently, the Child-Pugh score is widely accepted as a method to assess liver function during chronic liver disease, mainly cirrhosis $(3,4)$. The Child-Pugh scoring system employs five clinical measures; serum albumin and bilirubin, ascites, encephalopathy and prothrombin time (PT), while the etiology of cirrhosis is not considered. In other words, hepatic capacity of protein synthesis is regarded as an important aspect of the Child-Pugh scoring system, and the evaluation system works on the assumption that every parameter worsens in parallel according to the progression of liver fibrosis, irrespective of the etiology. However, in a previous report examining prediction factors for variceal hemorrhage, the form of varices, red color sign and alcoholism were independent risk factors, whereas Child-Pugh variables were not included as significant factors (5). The result indicates that for each cause of cirrhosis, the relationship between the degree of fibrosis and clinical findings, including the capacity for protein synthesis, may vary.

In our experience, certain cirrhotic patients unexpectedly show high serum levels of albumin despite advanced liver fibrosis. Certain patients with alcoholic cirrhosis who underwent liver resection for hepatocellular carcinoma (HCC) had severe post-operational liver failure, even though they were within the permissible range under pre-evaluation as Child-Pugh class A with more than $3.5 \mathrm{~g} / \mathrm{dl}$ of serum albumin. This is perhaps because the score for evaluating residual liver function was overestimated due to their serum albumin levels. Therefore, in this study we assessed hepatic protein synthesis 
Table I. Patient characteristics.

\begin{tabular}{|c|c|c|c|}
\hline & $\begin{array}{l}\text { Alcohol group } \\
\left(\text { alcohol }^{+} / \mathrm{HCV}^{-}\right)\end{array}$ & $\begin{array}{l}\text { HCV group } \\
\left(\text { alcohol }^{-} / \mathrm{HCV}^{+}\right)\end{array}$ & $\begin{array}{l}\text { Alcohol + HCV group } \\
\left(\text { alcohol }^{+} / \mathrm{HCV}^{+}\right)\end{array}$ \\
\hline No. of patients & 27 & 31 & 31 \\
\hline Age (years) & $67.2 \pm 7.6$ & $68.2 \pm 7.9$ & $64.2 \pm 9.7$ \\
\hline Gender (male/female) & $3 / 24$ & $4 / 27$ & $4 / 27$ \\
\hline $\operatorname{BMI}\left(\mathrm{kg} / \mathrm{cm}^{2}\right)$ & $23.6 \pm 0.5$ & $23.2 \pm 0.6$ & $23.4 \pm 0.6$ \\
\hline Albumin (g/dl) & $3.7 \pm 0.1$ & $3.2 \pm 0.1$ & $3.6 \pm 0.1^{\mathrm{a}}$ \\
\hline Bilirubin (mg/dl) & $1.8 \pm 0.2$ & $1.7 \pm 0.2$ & $1.3 \pm 0.2$ \\
\hline $\operatorname{AST}(\mathrm{U} / \mathrm{l})$ & $44.6 \pm 3.8^{c}$ & $73.1 \pm 5.9$ & $74.3 \pm 6.6$ \\
\hline ALT (U/l) & $27.3 \pm 1.9^{\mathrm{d}}$ & $57.6 \pm 5.3$ & $63.4 \pm 5.8$ \\
\hline LDH (U/l) & $292.8 \pm 25.2$ & $410.7 \pm 37.5$ & $356.1 \pm 30.2$ \\
\hline GGT (U/l) & $195.8 \pm 34.0^{\mathrm{b}}$ & $52.7 \pm 7.0$ & $103.7 \pm 15.0^{\mathrm{a}}$ \\
\hline ALP (U/l) & $390.3 \pm 41.3$ & $452.8 \pm 53.0$ & $433.7 \pm 44.9$ \\
\hline ChE (mg/dl) & $103.3 \pm 9.1^{\mathrm{b}}$ & $70.9 \pm 6.4$ & $81.3 \pm 5.4$ \\
\hline Cholesterol (mg/dl) & $159.3 \pm 5.5^{\mathrm{b}}$ & $136.3 \pm 4.7$ & $141.9 \pm 4.8$ \\
\hline $\mathrm{PT}(\%)$ & $64.7 \pm 4.2$ & $68.1 \pm 2.8$ & $66.2 \pm 4.1$ \\
\hline $\mathrm{WBC}(/ \mu \mathrm{l})$ & $4,444.8 \pm 245.8^{b}$ & $3,647.7 \pm 221.0$ & $3,637.7 \pm 199.4$ \\
\hline $\mathrm{RBC}(/ \mu \mathrm{l})$ & $392.6 \pm 12.5$ & $368.3 \pm 9.4$ & $373.7 \pm 10.0$ \\
\hline Hemoglobin (g/dl) & $12.5 \pm 0.4$ & $12.0 \pm 0.4$ & $12.5 \pm 0.32$ \\
\hline Platelet $(/ \mu 1)$ & $8.2 \pm 0.5$ & $7.3 \pm 0.4$ & $7.5 \pm 0.5$ \\
\hline
\end{tabular}

BMI, body mass index; AST, aspartate aminotransferase; ALT, alanine aminotransferase; LDH, lactate dehydrogenase; GGT, $\gamma$-glutamyl transpeptidase; ALP, alkaline phosphatase; ChE, cholinesterase; PT, prothrombin time; WBC, white blood cells; RBC, red blood cells. ${ }^{\mathrm{P}} \mathrm{P}<0.01 \mathrm{vs}$. HCV group; ${ }^{\mathrm{b}} \mathrm{P}<0.05 ;{ }^{\mathrm{c}} \mathrm{P}<0.01 ;{ }^{\mathrm{d}} \mathrm{P}<0.001 \mathrm{vs}$. alcohol $+\mathrm{HCV}$ group.

capacity, including serum albumin levels, in patients with alcoholic cirrhosis and compared them to those in hepatitis $\mathrm{C}$ virus (HCV) infection-induced cirrhotic patients, which is the most common cause of liver cirrhosis and HCC. Platelet count was employed as a marker for liver fibrosis, since it has been previously used for predicting the progression of fibrosis $(6,7)$ and can be measured simply and non-invasively.

\section{Materials and methods}

From April 2000 to March 2006, 225 outpatients with compensated liver cirrhosis were seen at the Department of Hepatology and Pancreatology, Kyushu University Hospital, including $170 \mathrm{HCV}$-positive patients. We defined an alcoholic patient as one with a daily consumption of $>80 \mathrm{~g}$ ethanol for at least 10 years. Prior to enrolling the patients, patients with HCC, uncontrolled diabetes (HbAlc $>6.5 \%$ ) or recent appetite loss within 1 month were excluded. Furthermore, patients who had a splenal longitudinal size of $>15 \mathrm{~cm}$ were also excluded. This selection was required to minimize the influence of malnutrition and extrahepatic destruction of platelets. After the selection, 45 alcoholic patients and 88 non-drinkers were identified as HCV-positive patients, while 30 alcoholic patients and 12 non-drinkers were identified as HCV-negative patients. For the alcoholic patients, only those who ceased drinking for at least 2 months were enrolled.

Finally, 31 alcoholic patients with HCV infection were enrolled (alcohol + HCV group). Stratification of patients according to their platelet count showed that the counts were $<5.0 \times 10^{4} / \mu 1$ in 4 patients, $4.9-6.4 \times 10^{4} / \mu 1$ in 7 patients,
$6.5-7.9 \times 10^{4} / \mu 1$ in 7 patients, $8.0-9.4 \times 10^{4} / \mu 1$ in 6 patients, $9.5-10.9 \times 10^{4} / \mu 1$ in 3 patients and $>11.0 \times 10^{4} / \mu 1$ in 4 patients. To set a similar background to that of the alcohol + HCV group, we randomly selected 31 age-, gender-, body mass index (BMI)- and platelet count-matched non-drinking HCV-positive patients (HCV group). Similarly, we enrolled $27 \mathrm{HCV}$-negative alcoholic patients (alcohol group). All enrolled patients were negative for hepatitis B virus, anti-nuclear antibody and antimitochondrial antibody. The study protocol was approved by the Ethics Committee of the Kyushu University Hospital.

All quantitative data are expressed as the means \pm standard deviation. Differences between categorical variables were analyzed using the Chi-square test. The Student's t-test was used for continuous variables. We considered P-values $<0.05$ to denote statistical significance.

\section{Results}

Regarding the blood testing results, significant differences were found only for serum $\gamma$-glutamyl transpeptidase (GGT) and albumin levels between the $\mathrm{HCV}$ and alcohol $+\mathrm{HCV}$ groups (Table I). There were no significant differences between the groups in terms of serum cholinesterase (ChE) and PT, which are general indices of hepatic protein synthesis capacity as well as albumin. In the alcohol + HCV group, aspartate aminotransferase and alanine aminotransferase levels were significantly lower, and the levels of GGT, ChE, cholesterol and white blood cell counts were significantly higher compared to the alcohol group. Platelet counts had been adjusted among the three groups. 

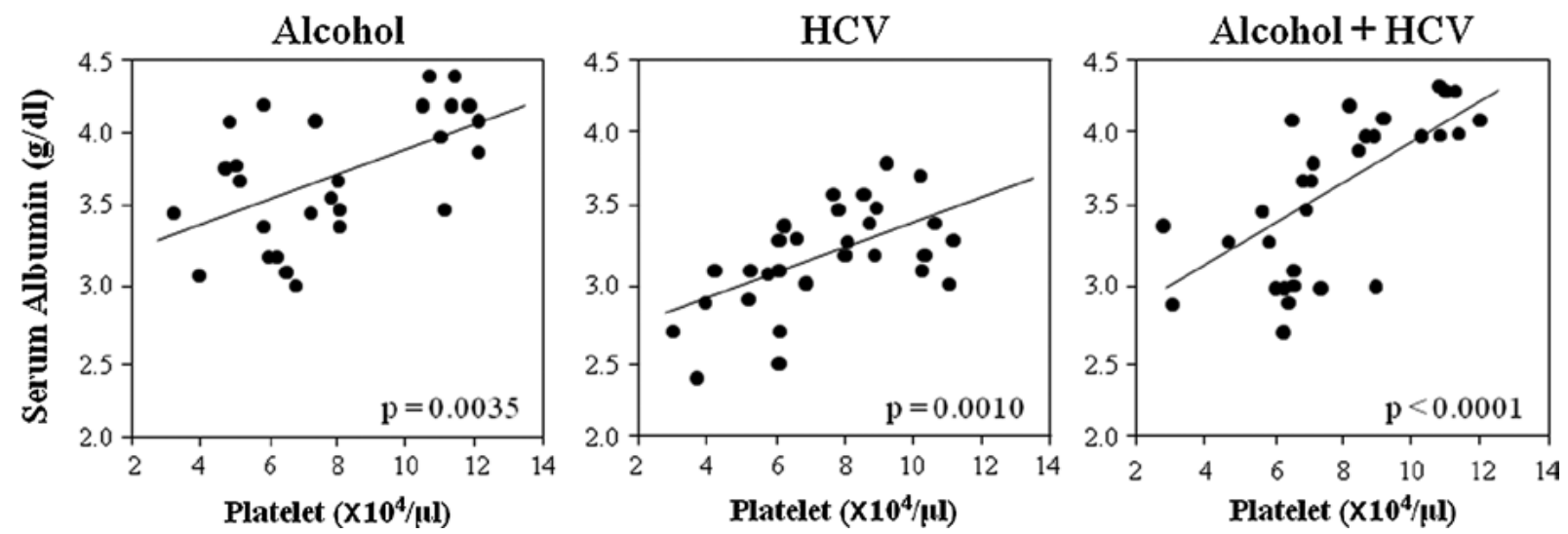

Figure 1. Correlation between serum albumin levels and platelet counts. Although the correlation was significant in each group, the levels of albumin in the alcohol group were always higher than in the HCV group. The approximate correlation line of the alcohol $+\mathrm{HCV}$ group was intermediate between the alcohol and HCV groups.
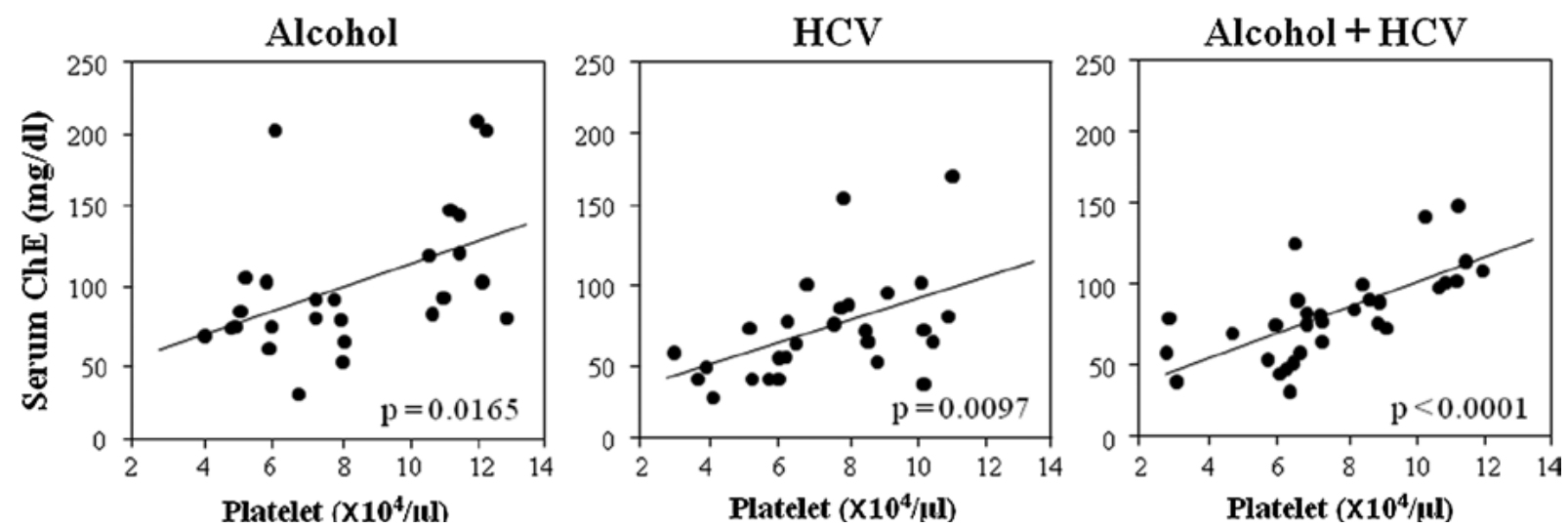

Figure 2. Correlation between serum cholinesterase levels and platelet counts. The correlations were almost similar in all three groups

To clarify the relationship between the degree of liver fibrosis and capacity for protein synthesis, the correlation between serum albumin levels and platelet counts in the three groups was examined (Fig. 1). In each group, albumin levels significantly correlated with platelet counts. However, albumin levels were always higher in the alcohol group than in the HCV group, and the difference was $\sim 0.5 \mathrm{~g} / \mathrm{dl}$ irrespective of the platelet count. The approximate correlation line of the alcohol + HCV group was intermediate between that of the alcohol and HCV groups.

To determine whether the relationship of serum albumin with platelet counts was also true between other proteins produced in the liver and platelet counts, we examined the correlation between serum $\mathrm{ChE}$ and platelet counts in each group (Fig. 2). The correlation between ChE and platelet counts was significant but, interestingly, the levels and slope of the approximate correlation lines were almost similar among the three groups, in contrast to the relationship between albumin and platelet counts.

\section{Discussion}

This study showed that serum albumin levels were higher in patients with alcoholic cirrhosis than in those with cirrhosis caused by HCV irrespective of platelet count. These findings indicate that the hepatic capacity of albumin synthesis may be affected by the etiology, alcohol or HCV, in addition to the degree of liver fibrosis. Since protein synthesis is also influenced by nutritional state (8), careful establishment of the conditions for enrolling patients was required. We excluded patients with HCC, uncontrolled diabetes or appetite loss. We also confirmed that the BMI distribution did not differ between the groups. Although we used platelet count as a marker of the degree of liver fibrosis, other methods, such as indocyanine green (ICG) tests, hyaluronic acid and pathological findings based on liver biopsy, can also be used. Whichever parameter is used as a liver fibrosis marker, it should be recognized that each has potential weaknesses; for example, a decrease in platelet count can be overestimated in patients with marked splenomegaly (9), ICG tests show higher values when a portosystemic shunt exists (10), serum hyaluronic acid levels cannot differentiate fibrotic stage F1-3 (11) and liver biopsies are invasive and associated with a risk of sampling error $(12,13)$. We employed the platelet count as it is a simple and non-invasive test, and is considerably reliable in patients at an advanced fibrotic stage (14). Patients with enlarged spleens (long axis diameter $>15 \mathrm{~cm}$ ) were excluded to reduce the influence of factors other than liver fibrosis and to increase its accuracy. Under these conditions, platelet counts probably reflect the degree of liver fibrosis, an assumption supported by the fact that platelet counts correlated significantly with serum albumin and ChE levels in all of the groups. 
It is noteworthy that PT and serum ChE levels did not differ and the relationship between serum $\mathrm{ChE}$ and the platelet count was almost similar among the three groups, indicating that, irrespective of the etiology, hepatocytic products other than albumin decrease equally according to the progression of liver fibrosis. Practically, the evaluation of the hepatic functional reserve of the patients may not yield reliable results if only albumin values are considered and the etiology of cirrhosis is ignored. Our results raise questions as to why patients with alcoholic cirrhosis would have higher serum albumin levels than those with cirrhosis caused by $\mathrm{HCV}$-infection. The role of $\mathrm{HCV}$ itself can be excluded as a significant difference was observed in albumin levels between the HCV and alcohol + HCV groups. Previous studies have suggested that alcohol can directly influence albumin synthesis. Annoni et al reported that patients with alcoholic cirrhosis showed significantly higher hepatic albumin mRNA levels than patients with a similar histological degree of cirrhosis due to viral infection (15). However, the alcoholic patients enrolled in the present study had stopped drinking at least 2 months prior to evaluation. Therefore, the possibility that alcohol or its metabolic products directly influenced the expression of albumin mRNA may be questionable. Other pathological differences may have contributed to the disparity in albumin synthesis, for example, the distinctive pathological characteristics of alcoholic cirrhosis, such as pericellular and perivenular fibrosis, Mallory bodies, steatosis and micronodular regeneration. Further investigations are required to determine the effect of these pathologies on albumin synthesis.

The present study demonstrated that the capacity for hepatic albumin synthesis in cirrhotic patients was differentially affected by the etiology of alcohol. Since serum albumin levels are commonly used as an important marker of hepatic functional reserve, ignoring the etiology of cirrhosis may lead to an incorrect evaluation. Serum albumin levels were present at higher levels and Child-Pugh scoring is likely to overestimate the residual hepatic functional reserve in patients with alcoholic cirrhosis. Therefore, alcohol consumption should be carefully considered when evaluating hepatic functional reserve.

\section{References}

1. Di Sario A, Feliciangeli G, Bendia E and Benedetti A: Diagnosis of liver fibrosis. Eur Rev Med Pharmacol Sci 8: 11-18, 2004.

2. Mullin EJ, Metcalfe MS and Maddern GJ: How much liver resection is too much? Am J Surg 190: 87-97, 2005.

3. Fazakas J, Mandli T, Ther G, et al: Evaluation of liver function for hepatic resection. Transplant Proc 38: 798-800, 2006.

4. Schneider PD: Preoperative assessment of liver function. Surg Clin North Am 84: 355-373, 2004.

5. Park DK, Um SH, Lee JW, et al: Clinical significance of variceal hemorrhage in recent years in patients with liver cirrhosis and esophageal varices. J Gastroenterol Hepatol 19: 1042-1051, 2004.

6. Forns X, Ampurdanès S, Llovet JM, et al: Identification of chronic hepatitis $\mathrm{C}$ patients without hepatic fibrosis by a simple predictive model. Hepatology 36: 986-992, 2002.

7. Wai CT, Greenson JK, Fontana RJ, Kalbfieisch JD, Marrero JA, Conjeevaram HS and Lok AS: A simple noninvasive index can predict both significant fibrosis and cirrhosis in patients with chronic hepatitis C. Hepatology 38: 518-516, 2003.

8. Manguso F, D'Ambra G, Menchise A, Sollazzo R and D'Agostino L: Effects of an appropriate oral diet on the nutritional status of patients with $\mathrm{HCV}$-related liver cirrhosis: a prospective study. Clin Nutr 24: 751-759, 2005.

9. Wadenvik H, Denfors I and Kutti J: Splenic blood flow and intrasplenic platelet kinetics in relation to spleen volume. Br J Haematol 67: 181-185, 1987.

10. Izuno K, Fujiyama S, Shibata J, Yoshida K, Sato T, Shimomura O and Takahashi M: Transrectal portal scintigraphy with I123 iodoamphetamine in liver diseases. Hepatogastroenterology 38: S8-S11, 1991.

11. Tatsumi C, Kudo M, Ueshima K, et al: Noninvasive evaluation of hepatic fibrosis using serum fibrotic markers, transient elastography (FibroScan) and real-time tissue elastography. Intervirology 51: 27-33, 2008 .

12. Poniachik J, Bernstein DE, Reddy KR, Jeffers LJ, Coelho-Little ME, Civantos F and Schiff ER: The role of laparoscopy in the diagnosis of cirrhosis. Gastrointest Endosc 43: 568-571, 1996.

13. Regev A, Berho M, Jeffers LJ, et al: Sampling error and intraobserver variation in liver biopsy in patients with chronic $\mathrm{HCV}$ infection. Am J Gastroenterol 97: 2614-2618, 2002.

14. Fontana RJ, Goodman ZG, Dienstag JL, et al: Relationship of serum fibrosis markers with liver fibrosis stage and collagen content in patients with advanced chronic hepatitis C. Hepatology 47: 789-798, 2008.

15. Annoni G, Weiner FR, Colombo M, Czaja MJ and Zern MA: Albumin and collagen gene regulation in alcohol- and virusinduced human liver disease. Gastroenterology 98: 197-202, 1990. 